\title{
Plasmon-Enhanced Photoluminescence of an Amorphous Silicon Quantum Dot Light-Emitting Device by Localized Surface Plasmon Polaritons in Ag/SiO ${ }_{x}: \mathbf{a}-\mathrm{Si}$ QDs/Ag Sandwich Nanostructures
}

\author{
Tsung-Han Tsai, Ming-Yi Lin, Wing-Kit Choi, and Hoang Yan Lin \\ Graduate Institute of Photonics and Optoelectronics and Department of Electrical Engineering, National Taiwan University, \\ No. 1, Section 4, Roosevelt Road, Taipei 106, Taiwan \\ Correspondence should be addressed to Hoang Yan Lin; hoangyanlin@ntu.edu.tw
}

Received 19 December 2014; Accepted 30 March 2015

Academic Editor: Jun Zhu

Copyright (c) 2015 Tsung-Han Tsai et al. This is an open access article distributed under the Creative Commons Attribution License, which permits unrestricted use, distribution, and reproduction in any medium, provided the original work is properly cited.

We investigated experimentally the plasmon-enhanced photoluminescence of the amorphous silicon quantum dots (a-Si QDs) light-emitting devices (LEDs) with the $\mathrm{Ag} / \mathrm{SiO}$ : $: \mathrm{a}-\mathrm{Si} \mathrm{QDs} / \mathrm{Ag}$ sandwich nanostructures, through the coupling between the a-Si QDs and localized surface plasmons polaritons (LSPPs) mode, by tuning a one-dimensional (1D) Ag grating on the top. The coupling of surface plasmons at the top and bottom $\mathrm{Ag} / \mathrm{SiO}_{x}: \mathrm{a}-\mathrm{Si}$ QDs interfaces resulted in the localized surface plasmon polaritons (LSPPs) confined underneath the Ag lines, which exhibit the Fabry-Pérot resonance. From the Raman spectrum, it proves the existence of a-Si QDs embedded in Si-rich $\mathrm{SiO}_{x}$ film $\left(\mathrm{SiO}_{x}\right.$ :a-Si QDs) at a low annealing temperature $\left(300^{\circ} \mathrm{C}\right)$ to prevent the possible diffusion of Ag atoms from Ag film. The photoluminescence (PL) spectra of a-Si QDs can be precisely tuned by a $1 \mathrm{D}$ Ag grating with different pitches and Ag line widths were investigated. An optimized Ag grating structure, with $500 \mathrm{~nm}$ pitch and $125 \mathrm{~nm}$ Ag line width, was found to achieve up to 4.8-fold PL enhancement at $526 \mathrm{~nm}$ and 2.46-fold PL integrated intensity compared to the a-Si QDs LEDs without Ag grating structure, due to the strong a-Si QDs-LSPPs coupling.

\section{Introduction}

Silicon quantum dots (Si QDs) light-emitting devices (LEDs) have been intensively investigated as a promising light source in recent years, for the next generation of Si-based optoelectronic integrated circuits (OEICs) [1-4]. The advantage of Si QDs LEDs lies in the compatible fabrication process with complementary metal-oxide-semiconductor (CMOS) and the low cost fabrication. However, realizing practical applications for Si QDs LEDs in OEICs requires high emission intensity, narrow spectral band, and low-temperature synthesis of Si QDs. Recently, surface plasmons (SPs), both surface plasmon polaritons (SPPs) and localized surface plasmon polaritons (LSPPs), have attracted a great deal of attention for their significant enhancements of photoluminescence (PL) intensity by coupling Si QDs into the near-field of SPs [58]. Meanwhile, the modified electromagnetic response of SPs in metal-insulator-metal (MIM) sandwich nanostructures through the coupling of SPs has been widely studied [9-16]. The coupling interaction via near-fields strongly depends on the thickness of the insulator and the structure parameter of texturing metallic surfaces. According to Fermi's golden rule, the electrical field intensity and the density of states of the LSPPs mode at the emitter position are directly related to the radiative recombination rate $\left(\Gamma_{\text {rad }}\right)$ for exciton dipoles of a-Si QDs $[17,18]$ :

$$
\Gamma_{\mathrm{rad}}=\frac{2 \pi}{\hbar}|\langle f|\vec{\mu} \cdot \vec{E}| i\rangle|^{2} \rho(\omega)
$$

where $\mu$ denotes the exciton dipole moment of a-Si QDs, $E$ is the electrical field intensity, and $\rho(\omega)$ is the density of states of the LSPPs mode. The radiative recombination rate of a-Si QDs can be greatly enhanced by a-Si QDs-LSPPs coupling, resulting in an increase in the PL intensity of a-Si QDs. Although the enhancement of PL intensity of crystalline Si QDs (c-Si QDs) has been reported through SPs coupling 


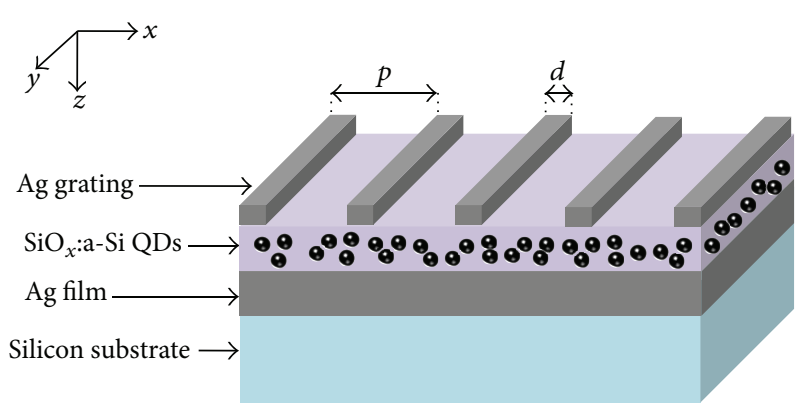

FIGURE 1: Schematic view of a-Si QDs LEDs with a $\mathrm{Ag} / \mathrm{SiO}_{x}: \mathrm{a}-\mathrm{Si}$ QDs/Ag sandwich nanostructures.

TABLE 1: The structural parameters of samples A-E, and the Ag grating with Ag line width $d$ and pitch $p$ on the $\mathrm{SiO}_{x}$ :a-Si QDs film. Sample A is the reference sample without Ag grating structure.

\begin{tabular}{lccc}
\hline Sample & $d$ & $p$ & $d / p$ \\
\hline A & - & - & - \\
B & $100 \mathrm{~nm}$ & $400 \mathrm{~nm}$ & 0.25 \\
C & $125 \mathrm{~nm}$ & $500 \mathrm{~nm}$ & 0.25 \\
D & $150 \mathrm{~nm}$ & $600 \mathrm{~nm}$ & 0.25 \\
E & $175 \mathrm{~nm}$ & $700 \mathrm{~nm}$ & 0.25 \\
\hline
\end{tabular}

effect [5-8], there has been no previous report concerning the enhanced PL intensity of a-Si QDs LEDs through a-Si QDsLSPPs coupling, within the MIM sandwich nanostructures. In this paper, we report the enhanced PL integrated intensity and the narrower full width at half-maximum (FWHM) of the PL spectra for a-Si QDs LEDs, with the $\mathrm{Ag} / \mathrm{SiO}_{x}: \mathrm{a}-$ $\mathrm{Si} \mathrm{QDs} / \mathrm{Ag}$ sandwich nanostructures relative to the a-Si LEDs without Ag grating, resulting from the strong coupling between a-Si QDs and LSPPs. The maximum 4.8-fold PL enhancement factor and $246 \%$ enhancement of PL integrated intensity have been observed, for an optimized Ag grating structure by a strong a-Si QDs-LSPPs coupling. We also prove the formation of a-Si QDs embedded in Si-rich $\mathrm{SiO}_{x}$ film $\left(\mathrm{SiO}_{x}: \mathrm{a}-\mathrm{Si} \mathrm{QDs}\right)$ with low annealing process $\left(300^{\circ} \mathrm{C}\right)$ and that the measured PL spectra of $\mathrm{SiO}_{x}: \mathrm{a}-\mathrm{Si}$ QDs originate from the quantum confinement effect (QCE) [19-22].

\section{Experiments}

The fabrication process of the trilayer $\mathrm{Ag} / \mathrm{SiO}_{x}: \mathrm{a}-\mathrm{Si} \mathrm{QDs} / \mathrm{Ag}$ sandwich nanostructures is described as follows. Silicon substrate was first coated with a $100 \mathrm{~nm}$-thick Ag film using thermal evaporation. Then, the $100 \mathrm{~nm}$-thick Si-rich $\mathrm{SiO}_{x}$ (SRO, $x<2$ ) film was deposited on the Ag film by using plasma enhanced chemical vapor deposition (PECVD) system at the pressure of $67 \mathrm{~Pa}$ with nitrogen-diluted $5 \% \mathrm{SiH}_{4}$ and $\mathrm{N}_{2} \mathrm{O}$ as the reactant gas sources. The flow rate of $\mathrm{N}_{2} \mathrm{O}$ gas was maintained at $30 \mathrm{sccm}$ and the $\mathrm{SiH}_{4} / \mathrm{N}_{2} \mathrm{O}$ flow rate ratio of $5.53: 1$. The sample was heated to $350^{\circ} \mathrm{C}$ and the radio frequency power was kept at $30 \mathrm{~W}$ during the SRO film growth. After the deposition, the SRO film was annealed at $300^{\circ} \mathrm{C}$ for $1 \mathrm{hr}$ in a quartz furnace with flowing $\mathrm{N}_{2}$ gas, to form a $\mathrm{SiO}_{x}$ :a$\mathrm{Si}$ QDs film. Then, a 1D periodic Ag grating was fabricated on the top of $\mathrm{SiO}_{x}$ :a-Si QDs film using electron-beam (e-beam) lithography (Elionix ELS-7500) and liftoff process. First, a $300 \mathrm{~nm}$-thick positive-type e-beam resist (Nippon Zeon ZEP$520 \mathrm{~A}$ ) is spun on the top of sample followed by the subsequent e-beam lithography to define the grating pattern with the pitch $p$ and line width $d$. The duty cycle $(d / p)$ of Ag grating is fixed at $25 \%$. Second, a $50 \mathrm{~nm}$-thick Ag film is deposited onto the patterned e-beam resist and the $\mathrm{Ag}$ grating is fabricated by liftoff process in an exclusive remover (Nippon Zeon ZDMAC). Figure 1 shows the schematic representation of device with $\mathrm{Ag} / \mathrm{SiO}_{x}$ :a-Si QDs/Ag sandwich nanostructures. The structural parameters of devices (samples A-E) are listed in Table 1. Scanning electron microscopy (SEM) images of Ag gratings (samples B-E) are shown in Figure 2. The room-temperature photoluminescence $(\mathrm{PL})$ spectra were acquired under the excitation from a $\mathrm{He}-\mathrm{Cd}$ laser operating at $\lambda_{\text {excitation }}=325 \mathrm{~nm}$ and with an average power of $50 \mathrm{~mW}$. The PL intensity within $350 \mathrm{~nm}$ and $750 \mathrm{~nm}$ was recorded using a monochromator (CVI DK240) in conjunction with a photomultiplier (Hamamatsu R928) and a digital multimeter (HP 34401A). The reflection spectra were measured in an optical microscope consisting of a monochromator (Horiba Jobin Yvon iHR 320) and a broadband halogen lamp as a white light source incident through a 20x objective $(\mathrm{NA}=$ $0.75)$ to the normal of the metal surface ( $z$-axis). And the reflected light was collected with the same objective. The refraction index of Si-rich $\mathrm{SiO}_{x}$ film was determined by an ellipsometer (JA Woollam M-2000DI). The thickness of each Ag film and $\mathrm{SiO}_{x}$ :a-Si QDs film was measured by an atomic force microscope (AFM, Veeco D5000). The Si/O composition ratio and concentration-depth profile of SRO film were measured by X-ray photoelectron spectroscopy (XPS, Thermo Fisher Scientific Theta probe). The existence and the size of a-Si QDs were measured by a Raman spectroscopy (Horiba Jobin Yvon T64000).

\section{Results and Discussions}

3.1. Material Analysis of $\mathrm{SiO}_{x}$ :a-Si QDs Film. Figure 3(a) shows that the concentration-depth profiles of the $\mathrm{SiO}_{x}$ :aSi QDs film were performed by using $\mathrm{Si} 2 p, \mathrm{O} 1 s$, and $\mathrm{Ag}$ $3 d$ peaks from XPS analysis. The average $\mathrm{Si}$ concentration of the $\mathrm{SiO}_{x}: \mathrm{a}-\mathrm{Si}$ QDs film is about 48.27 at. \%. High $\mathrm{Si} / \mathrm{O}$ composition ratio for the $\mathrm{SiO}_{x}$ :a-Si QDs film is observed, due to the high $\mathrm{SiH}_{4} / \mathrm{N}_{2} \mathrm{O}$ flow rate ratio of 5.53:1 during the PECVD growth. Since there are excessive $\mathrm{Si}$ atoms and insufficient $\mathrm{O}$ atoms, the $\mathrm{Si}$ atoms could move simply and accumulate to form a-Si QDs, without being restrained by Si$\mathrm{O}$ bonds of the $\mathrm{SiO}_{x}$ film during the annealing process [23, $24]$. Hence, we conclude that the a-Si QDs can be synthesized at a low annealing temperature $\left(300^{\circ} \mathrm{C}\right)$. The $\mathrm{Ag}$ atoms did not diffuse into the $\mathrm{SiO}_{x}$ :a-Si QDs film from bottom $\mathrm{Ag}$ film after annealing process, as shown in Figure 3(a). Raman spectroscopy was used to analyze the size of Si QDs through the energy shift of the Raman peak and the correspondent line broadening [25]. Figure 3(b) shows that the Raman spectrum of $\mathrm{SiO}_{x}$ :a-Si QDs film can be separated into two components. One component corresponds to the a-Si QDs, exhibiting a 

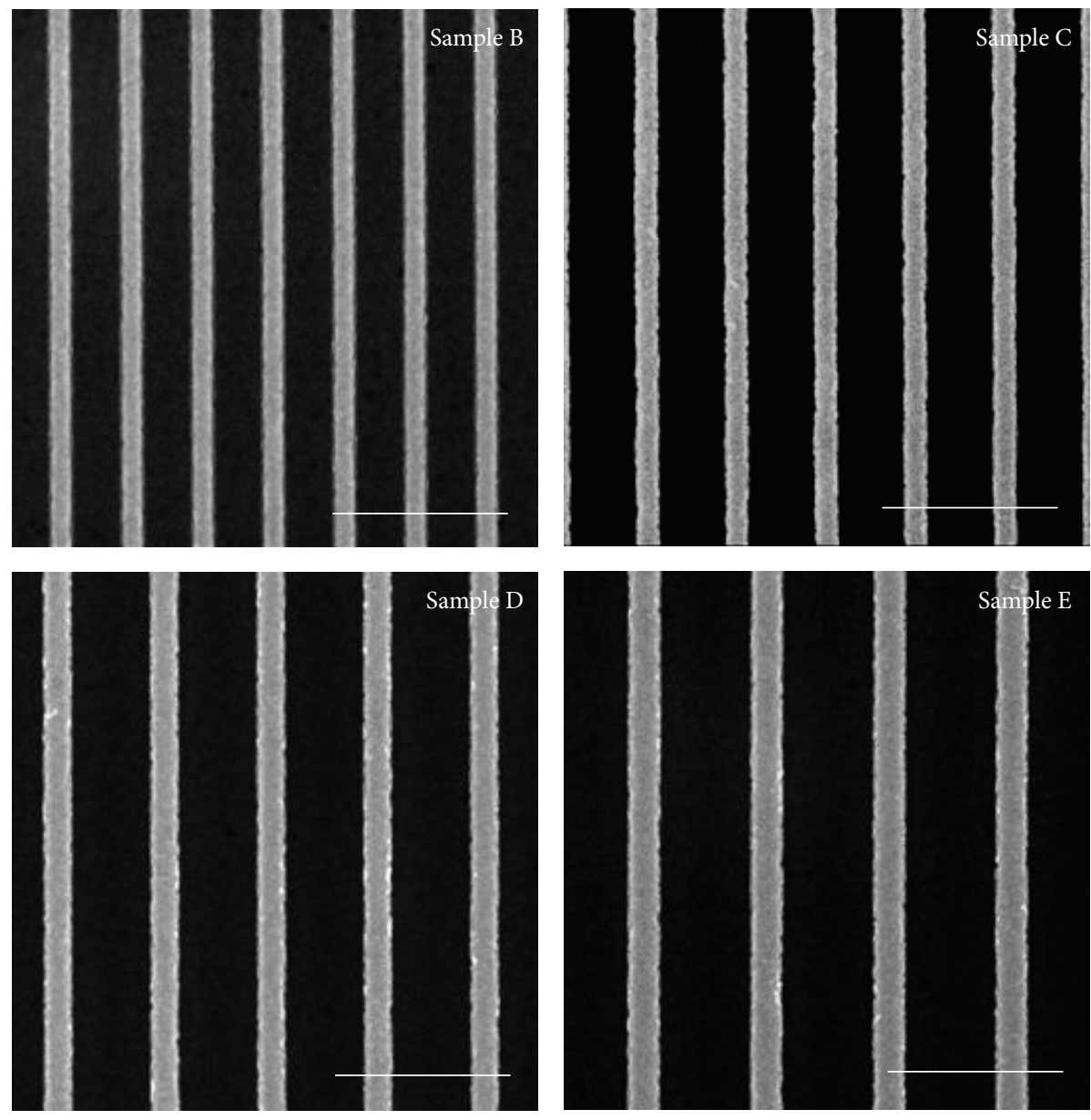

FIGURE 2: SEM images of a series of Ag gratings (samples B-E). Scale bar is $1 \mu \mathrm{m}$.

peak at $490 \mathrm{~cm}^{-1}$ with a FWHM of $33 \mathrm{~cm}^{-1}$. The Raman downshift and FWHM value show that the sizes of a-Si QDs are about $1.7 \mathrm{~nm}$. Figure 3(c) presents the room-temperature PL spectrum of a-Si QDs LEDs without Ag grating structure (sample A), showing that the center emission wavelength is about $510 \mathrm{~nm}$. The center emission wavelength of our $1.7 \mathrm{~nm}$ sized a-Si QDs shows a good agreement with the theoretical study of light emission properties of $\mathrm{SiO}_{x}$ :a-Si QDs film [20]. Hence, the existence of a-Si QDs embedded in the $\mathrm{SiO}_{x}$ matrix with the low annealing process $\left(300^{\circ} \mathrm{C}\right)$ is hereby proved by the Raman spectrum. Also, the main PL peak of a-Si QDs does not overlap with the PL spectrum from the oxygen related defects [26-28]. On the other hand, the smaller the a-Si QDs $(\sim 1.7 \mathrm{~nm})$ are, the stronger the QCE is to surpass the interface state recombination $[22,29]$. Hence, we conclude that the measured PL spectrum originates from the QCE of the a-Si QDs.

3.2. Optical Property Analysis (Sample A-D). The reflection spectra of samples B-E are shown in Figure 4 which exhibited reflection dips that can be attributed to the extinction due to the excitation of LSPPs confined at the top $\mathrm{Ag} / \mathrm{SiO}$ : $\mathrm{a}-\mathrm{Si}$ QDs interface. It is because when the $\mathrm{SiO}_{x}: \mathrm{a}-\mathrm{Si}$ QDs film is thin enough, the SPPs excited at the top Ag/SiO $:$ :a-Si QDs interface by TM-polarized light emitted by the a-Si QDs will couple with the SPPs at the bottom Ag/SiO :a-Si QDs interface via evanescent fields [11-16]. The strong coupling effect causes the excitation of LSPPs, which exhibits the Fabry-Pérot resonance in the $x$ direction between the two sidewalls of the Ag line [11-15]. It is found that the LSPPs resonances satisfy this equation:

$$
d \approx \frac{m \lambda}{2 n_{\mathrm{eff}}(\lambda)},
$$

where $d$ is the Ag line width, $\lambda$ is the resonance wavelength of the LSPPs (the wavelength of the minimum reflection), $n_{\text {eff }}$ is the effective refractive index of the $\mathrm{SiO}_{x}: \mathrm{a}-\mathrm{Si}$ QDs film at $\lambda$, and $m$ is an integer (LSPPs modes). The observed resonance wavelengths for each sample are $425 \mathrm{~nm}$ (sample $\mathrm{B}, m=1$ ), $526 \mathrm{~nm}$ (sample C, $m=1$ ), $629 \mathrm{~nm}$ (sample $\mathrm{D}$, $m=1$ ), $731 \mathrm{~nm}$, and $380 \mathrm{~nm}$ (sample E, $m=1$ and $m=$ 2 ), respectively. The LSPPs resonance wavelength increases with the Ag line width. The original refraction indices of the $\mathrm{SiO}_{x}$ :a-Si QDs film that were determined by ellipsometer at 425, 526, 629, 731, and $380 \mathrm{~nm}$ are $n=1.97,1.95,1.94,1.93$, and 1.99, respectively. In our $\mathrm{Ag} / \mathrm{SiO}_{x}: \mathrm{a}-\mathrm{Si} \mathrm{QDs} / \mathrm{Ag}$ sandwich nanostructures, the effective refraction index of $\mathrm{SiO}_{x}: \mathrm{a}-\mathrm{Si}$ QDs film increased to 1.076-1.083 times of the original values, 

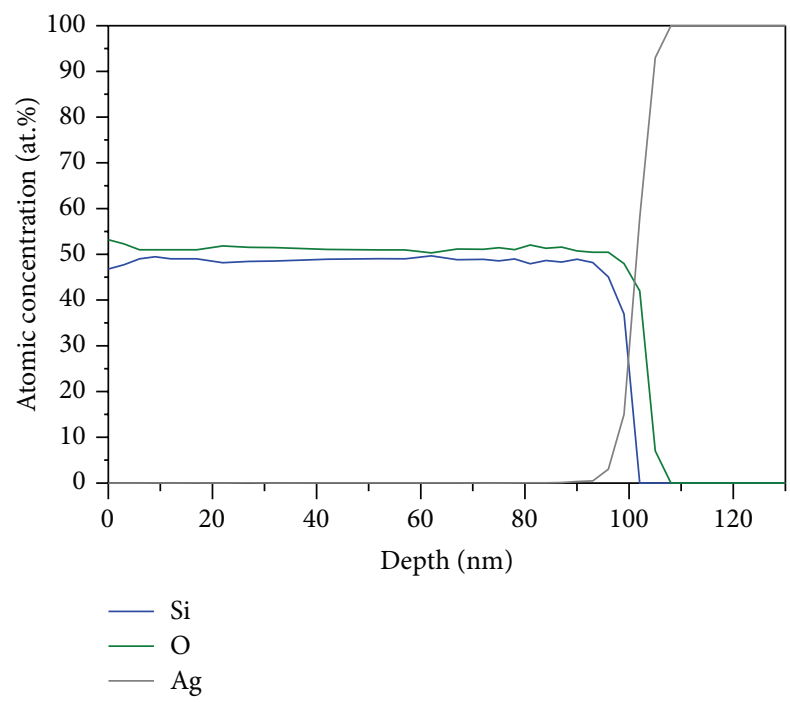

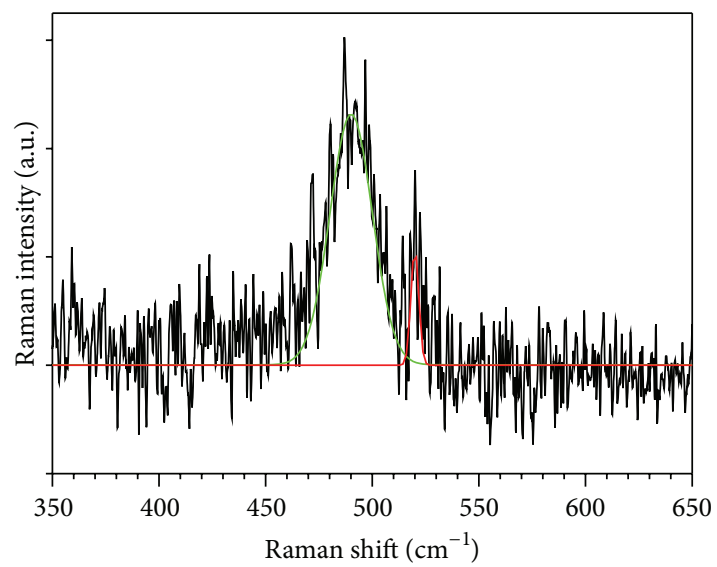

- Measured-curve Gaussian fit for a-Si QDs signal — Gaussian fit for Si substrate signal

(b)

(a)

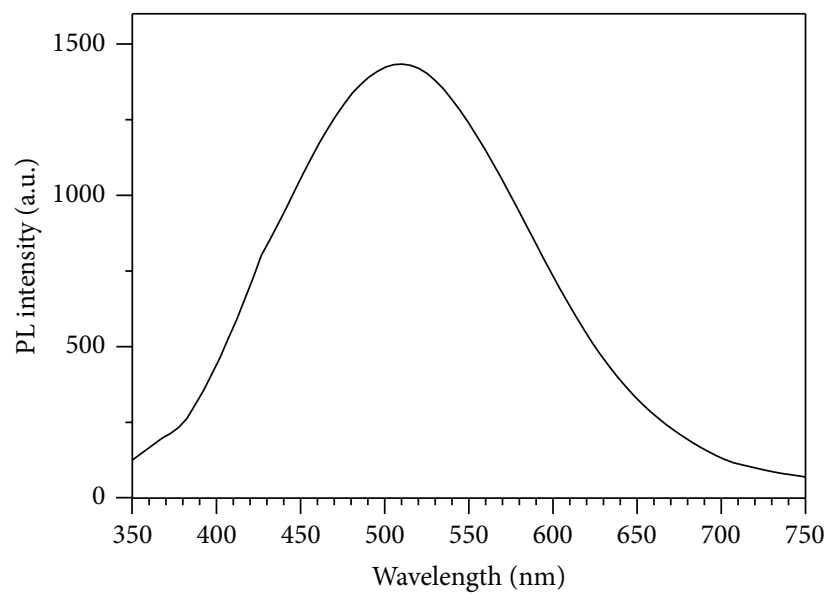

(c)

FIgure 3: (a) XPS depth profiles for $\mathrm{Si}, \mathrm{O}$, and Ag elements in $\mathrm{SiO}_{x}: \mathrm{a}-\mathrm{Si}$ QDs film and at $\mathrm{SiO}_{x}: \mathrm{a}-\mathrm{Si}$ QDs film/Ag film interface. (b) Raman spectrum of $\mathrm{SiO}_{x}: \mathrm{a}-\mathrm{Si}$ QDs film. The spectrum is separated into two components corresponding to the a-Si QDs and the Si substrate (by Gaussian fit). (c) PL spectrum of sample A.

due to the interaction between LSPPs and the induced image dipole (which has the opposite orientation) [30]. Figure 5 shows the measured room-temperature PL spectra of samples A-E. The PL integrated intensity of samples B-E has been enhanced compared to sample A as a reference sample by increasing the radiative recombination rate of a-Si QDs due to the coupling with LSPPs mode. According to Fermi's golden rule [18], when the exciton dipole moments $(\mu)$ of a-Si QDs strongly couple to the near-field of LSPPs, the radiative recombination rate of a-Si QDs can be enhanced by the large density of states of LSPPs, resulting in an increase in emission intensity. The largest enhancement of the PL integrated intensity reaches $246 \%$ and the narrowest FWHM of $67 \mathrm{~nm}$ for sample $\mathrm{C}$, due to the close match between the original center emission wavelength of a-Si QDs $(510 \mathrm{~nm}$ ) and the LSPPs resonance wavelength $(526 \mathrm{~nm})$. Sample C shows the strongest a-Si QDs-LSPPs coupling among samples
B-E. The mismatch between the original center emission wavelength of a-Si QDs and LSPPs resonance wavelength for samples B, D, and E results in not only the lower enhancement of PL integrated intensity and broadened FWHM than sample $\mathrm{C}$, but also the distorted emission spectra. Hence, the PL integrated intensities for samples B, D, and E are only enhanced by $172 \%, 161 \%$, and $132 \%$, respectively. For samples $\mathrm{B}, \mathrm{D}$, and $\mathrm{E}$, the multipeaks in the PL spectra observed in Figure 5 correspond to the original emission peak of a-Si QDs and the respective excited LSPPs modes as shown in Figure 4 . The phenomenon is attributed to the increase in the electric field intensity and the density of states of the LSPPs mode at the emitter position as the wavelength is near the LSPPs resonance, resulting in the enhancements of the radiative recombination rate and the emission intensity. Also, the main PL peaks of samples B, D, and E were shifted from the original center emission wavelength of a-Si QDs towards 


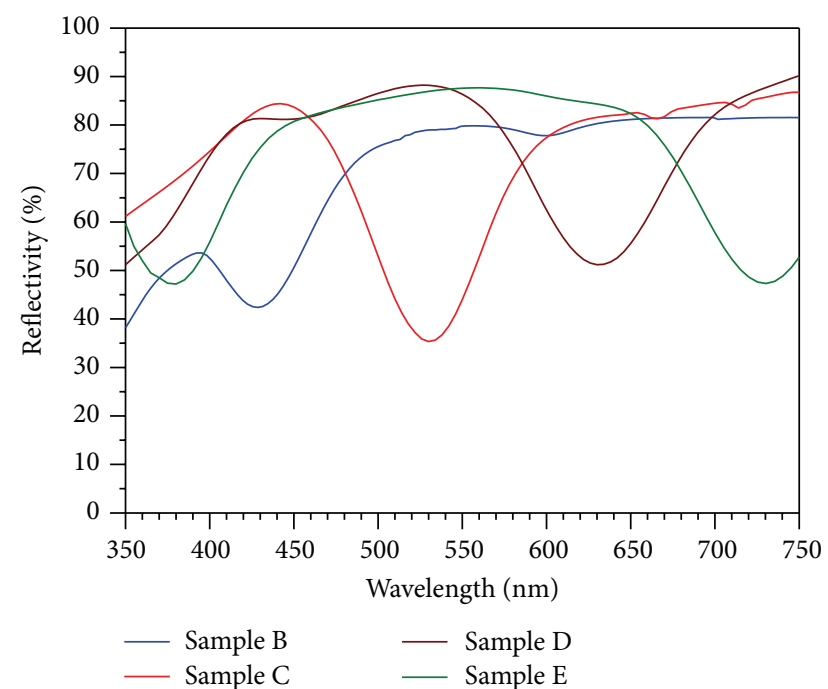

Figure 4: Reflection spectra of samples B-E.

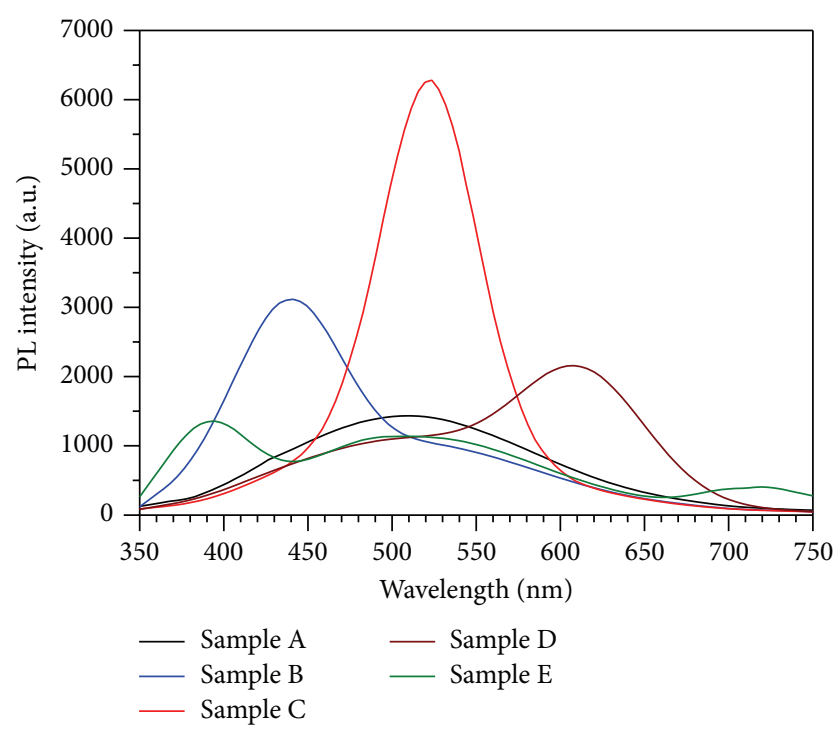

Figure 5: PL spectra of samples A-E.

the respective LSPPs resonance wavelengths. Figure 6 shows the plots of the PL enhancement factor, $I_{\text {grating }}(\lambda) / I_{\text {ref }}(\lambda)$, where $I_{\text {grating }}(\lambda)$ and $I_{\text {ref }}(\lambda)$ are the PL intensities for the samples with and without Ag grating. The plots of the PL enhancement factor show a redshift with an increase in the $\mathrm{Ag}$ line width that is similar to the tendency of reflection dips, and it indicates that the PL enhancement factor strongly corresponds with the LSPPs modes. From the results, a maximum PL enhancement factor of 4.8 was observed at $526 \mathrm{~nm}$ for sample $\mathrm{C}$ due to the strong a-Si QDs-LSPPs coupling. Therefore, it is worthwhile noticing the improved design of $\mathrm{Ag}$ grating structure by tuning the pitch and $\mathrm{Ag}$ line width for the largest PL integrated emission through the strong a-Si QDs-LSPPs coupling.

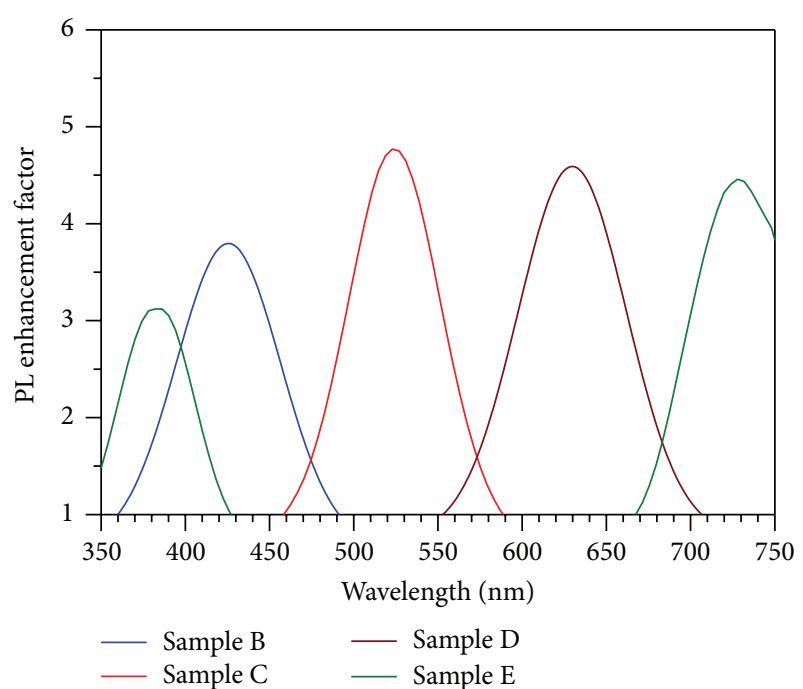

Figure 6: The plots of the PL enhancement factor of samples B-E.

\section{Conclusions}

In conclusion, we proposed the plasmon-enhanced PL intensity of a-Si QDs LEDs with $\mathrm{Ag} / \mathrm{SiO}_{x}$ :a-Si QDs/Ag sandwich nanostructures resulting from the strong coupling between a-Si QDs and LSPPs modes. It was found that the LSPPs were excited underneath the Ag lines, which exhibit the Fabry-Pérot resonance resulting from the coupling of SPPs between the top Ag grating and bottom $\mathrm{Ag}$ film. A narrowest $67 \mathrm{~nm}$ FWHM of PL spectrum, a maximum of 4.8-fold PL enhancement factor, and the largest 2.46-fold PL integrated intensity, compared to the a-Si QDs LEDs without Ag grating structure, have been observed for an optimized Ag grating structure by the strong a-Si QDs-LSPPs coupling.

\section{Conflict of Interests}

The authors declare that there is no conflict of interests regarding the publication of this paper.

\section{Acknowledgments}

The authors would like to acknowledge the Ministry of Science and Technology of Taiwan for the financial support of this research under Contracts nos. MOST 102-2221-E002-205-MY3 and MOST 104-3113-E-155-001 and National Taiwan University under the Aim for Top University Projects 104R7607-4 and 104R8908.

\section{References}

[1] D. A. B. Miller, "Silicon sees the light," Nature, vol. 378, no. 6554, p. 238, 1995.

[2] L. Pavesi, L. Dal Negro, C. Mazzoleni, G. Franzò, and F. Priolo, "Optical gain in silicon nanocrystals," Nature, vol. 408, no. 6811, pp. 440-444, 2000.

[3] P. M. Fauchet, "Light emission from Si quantum dots," Materials Today, vol. 8, no. 1, pp. 26-33, 2005. 
[4] G. Y. Sung, N.-M. Park, J.-H. Shin et al., "Physics and device structures of highly efficient silicon quantum dots based silicon nitride light-emitting diodes," IEEE Journal on Selected Topics in Quantum Electronics, vol. 12, no. 6, pp. 1545-1554, 2006.

[5] B.-H. Kim, C.-H. Cho, J.-S. Mun et al., "Enhancement of the external quantum efficiency of a silicon quantum dot light-emitting diode by localized surface plasmons," Advanced Materials, vol. 20, no. 16, pp. 3100-3104, 2008.

[6] J. S. Biteen, N. S. Lewis, H. A. Atwater, H. Mertens, and A. Polman, "Spectral tuning of plasmon-enhanced silicon quantum dot luminescence," Applied Physics Letters, vol. 88, no. 13, Article ID 131109, 2006.

[7] J. S. Biteen, L. A. Sweatlock, H. Mertens, N. S. Lewis, A. Polman, and H. A. Atwater, "Plasmon-enhanced photoluminescence of silicon quantum dots: simulation and experiment," The Journal of Physical Chemistry C, vol. 111, no. 36, pp. 13372-13377, 2007.

[8] H. Mertens, J. S. Biteen, H. A. Atwater, and A. Polman, "Polarization-selective plasmon-enhanced silicon quantum-dot luminescence," Nano Letters, vol. 6, no. 11, pp. 2622-2625, 2006.

[9] S. A. Maier, "Plasmonic field enhancement and SERS in the effective mode volume picture," Optics Express, vol. 14, no. 5, pp. 1957-1964, 2006.

[10] J.-J. Jiang, Y.-B. Xie, Z.-Y. Liu, X.-M. Tang, X.-J. Zhang, and Y.-Y. Zhu, "Amplified spontaneous emission via the coupling between Fabry-Perot cavity and surface plasmon polariton modes," Optics Letters, vol. 39, no. 8, pp. 2378-2381, 2014.

[11] Y.-H. Ye, Y.-W. Jiang, M.-W. Tsai et al., "Localized surface plasmon polaritons in $\mathrm{Ag} / \mathrm{SiO}_{2} / \mathrm{Ag}$ plasmonic thermal emitter," Applied Physics Letters, vol. 93, no. 3, Article ID 033113, 3 pages, 2008.

[12] Y.-H. Ye, Y.-W. Jiang, M.-W. Tsai et al., "Coupling of surface plasmons between two silver films in a $\mathrm{Ag} / \mathrm{SiO}_{2} / \mathrm{Ag}$ plasmonic thermal emitter with grating structure," Applied Physics Letters, vol. 93, no. 26, Article ID 263106, 2008.

[13] S. Collin, F. Pardo, and J.-L. Pelouard, "Waveguiding in nanoscale metallic apertures," Optics Express, vol. 15, no. 7, pp. 4310-4320, 2007.

[14] L. Martín-Moreno, F. J. García-Vidal, H. J. Lezec et al., “Theory of extraordinary optical transmission through subwavelength hole arrays," Physical Review Letters, vol. 86, article 1114, 2001.

[15] C.-M. Wang, Y.-C. Chang, M.-W. Tsai et al., "Reflection and emission properties of an infrared emitter," Optics Express, vol. 15, no. 22, pp. 14673-14678, 2007.

[16] S. A. Darmanyan and A. V. Zayats, "Light tunneling via resonant surface plasmon polariton states and the enhanced transmission of periodically nanostructured metal films: an analytical study," Physical Review B, vol. 67, no. 3, Article ID 035424, 7 pages, 2003.

[17] J. Kümmerlen, A. Leitner, H. Brunner, F. R. Aussenegg, and A. Wokaun, "Enhanced dye fluorescence over silver island films: analysis of the distance dependence," Molecular Physics, vol. 80, no. 5, pp. 1031-1046, 1993.

[18] E. Fermi, "Quantum theory of radiation," Reviews of Modern Physics, vol. 4, no. 1, pp. 87-132, 1932.

[19] N.-M. Park, C.-J. Choi, T.-Y. Seong, and S.-J. Park, “Quantum confinement in amorphous silicon quantum dots embedded in silicon nitride," Physical Review Letters, vol. 86, no. 7, pp. 13551357, 2001.

[20] K. Nishio, J. Kōga, T. Yamaguchi, and F. Yonezawa, "Theoretical study of light-emission properties of amorphous silicon quantum dots," Physical Review B, vol. 67, Article ID 195304, 2003.
[21] G. G. Qin and Y. J. Li, "Photoluminescence mechanism model for oxidized porous silicon and nanoscale-silicon-particleembedded silicon oxide," Physical Review B, vol. 68, no. 8, Article ID 085309, 7 pages, 2003.

[22] C. Delerue, G. Allan, and M. Lannoo, "Theoretical aspects of the luminescence of porous silicon," Physical Review B, vol. 48, no. 15, pp. 11024-11036, 1993.

[23] S. Mirabella, R. Agosta, G. Franzó et al., "Light absorption in silicon quantum dots embedded in silica," Journal of Applied Physics, vol. 106, no. 10, Article ID 103505, 2009.

[24] B.-H. Lai, C.-H. Cheng, Y.-H. Pai, and G.-R. Lin, "Plasma power controlled deposition of $\mathrm{SiO}_{x}$ with manipulated Si quantum dot size for photoluminescent wavelength tailoring," Optics Express, vol. 18, no. 5, pp. 4449-4456, 2010.

[25] G. Faraci, S. Gibilisco, P. Russo et al., " $\mathrm{Si} / \mathrm{SiO}_{2}$ core shell clusters probed by Raman spectroscopy," The European Physical Journal $B$-Condensed Matter and Complex Systems, vol. 46, no. 4, pp. 457-461, 2005.

[26] G. H. Li, K. Ding, Y. Chen, H. X. Han, and Z. P. Wang, "Photoluminescence and Raman scattering of silicon nanocrystals prepared," Journal of Applied Physics, vol. 88, no. 3, pp. 14391442, 2000.

[27] G.-R. Lin, C.-J. Lin, C.-K. Lin, L.-J. Chou, and Y.-L. Chueh, "Oxygen defect and Si nanocrystal dependent white-light and near-infrared electroluminescence of Si-implanted and plasmaenhanced chemical-vapor deposition-grown $\mathrm{Si}$-rich $\mathrm{SiO}_{2}$," Journal of Applied Physics, vol. 97, no. 9, Article ID 094306, 8 pages, 2005.

[28] G.-R. Lin, C.-J. Lin, and K.-C. Yu, "Time-resolved photoluminescence and capacitance-voltage analysis of the neutral vacancy defect in silicon implanted $\mathrm{SiO}_{2}$ on silicon substrate," Journal of Applied Physics, vol. 96, no. 5, article 3025, 3 pages, 2004.

[29] X. X. Wang, J. G. Zhang, L. Ding et al., "Origin and evolution of photoluminescence from $\mathrm{Si}$ nanocrystals embedded in a $\mathrm{SiO}_{2}$ matrix," Physical Review B, vol. 72, Article ID 195313, 2005.

[30] A. Christ, G. Lévêque, O. J. Martin et al., "Near-field-induced tunability of surface plasmon polaritons in composite metallic nanostructures," Journal of Microscopy, vol. 229, no. 2, pp. 344353, 2008. 

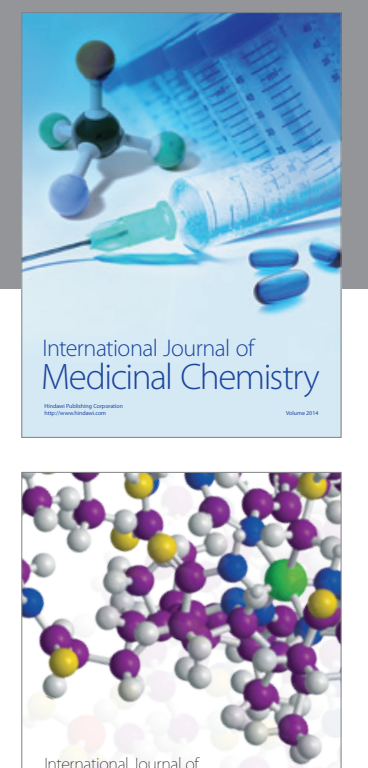

\section{Carbohydrate} Chemistry

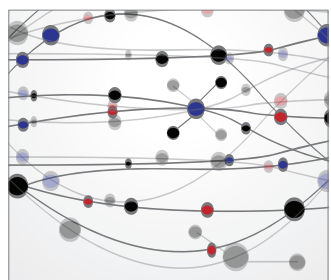

The Scientific World Journal
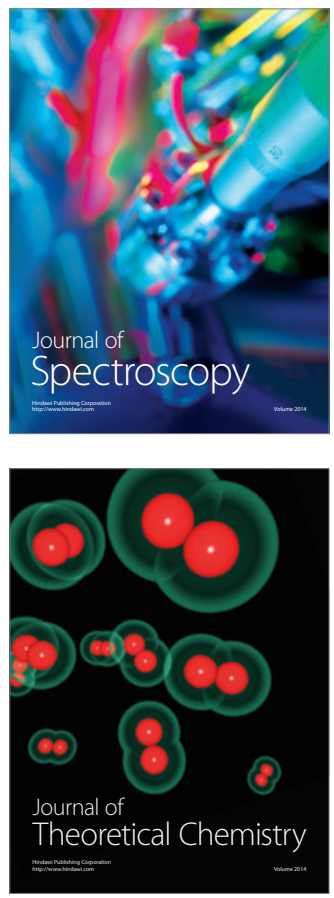
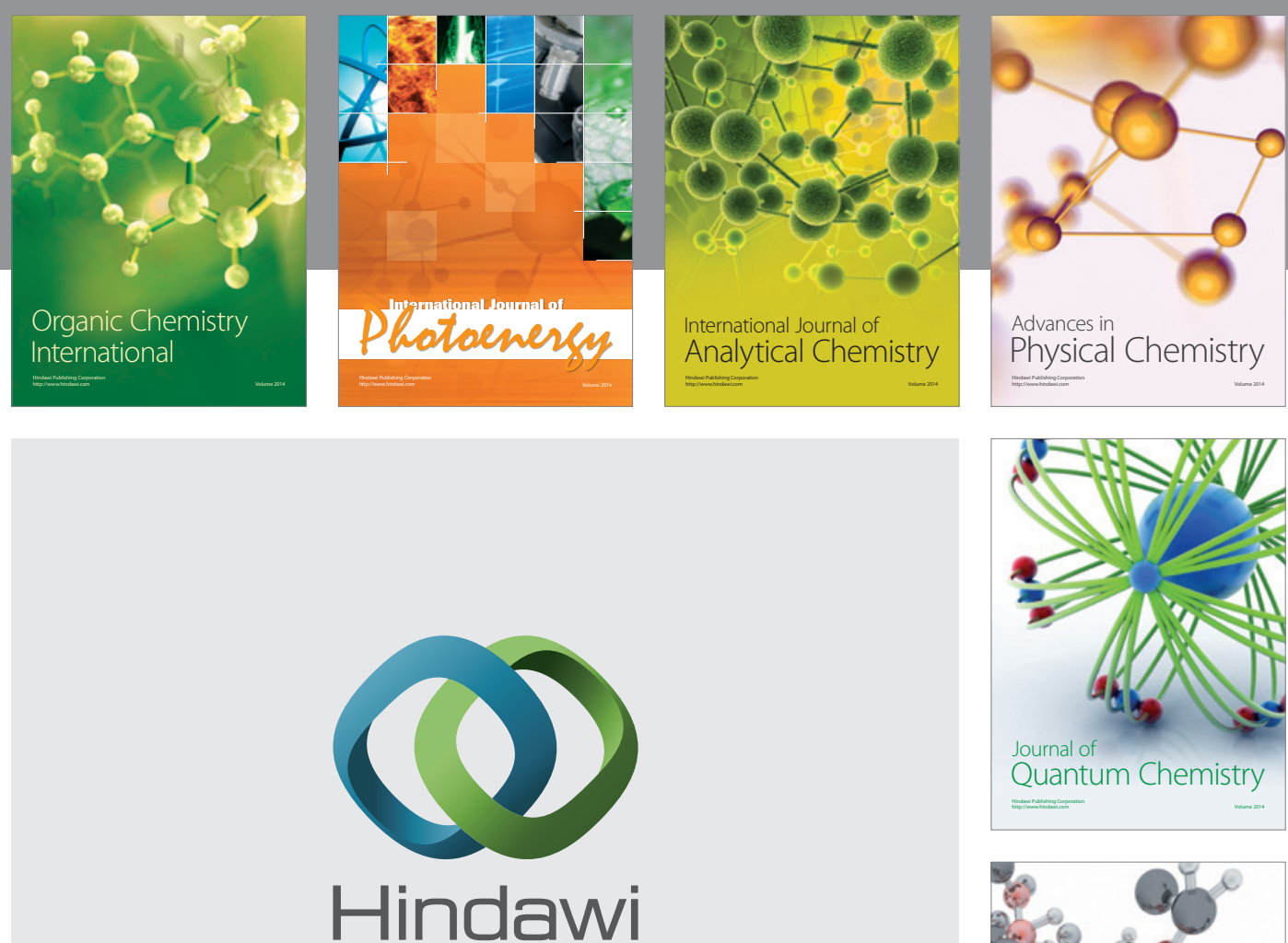

Submit your manuscripts at

http://www.hindawi.com

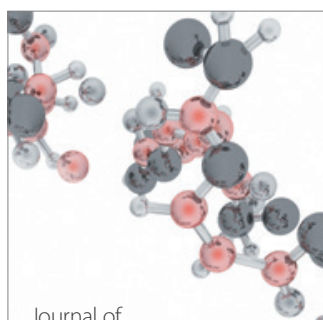

Analytical Methods

in Chemistry

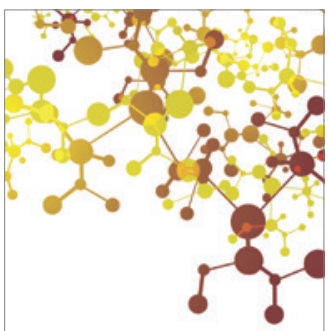

Journal of

Applied Chemistry

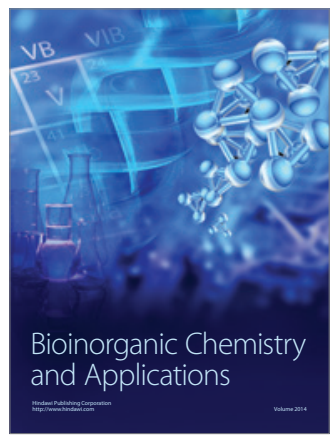

Inorganic Chemistry
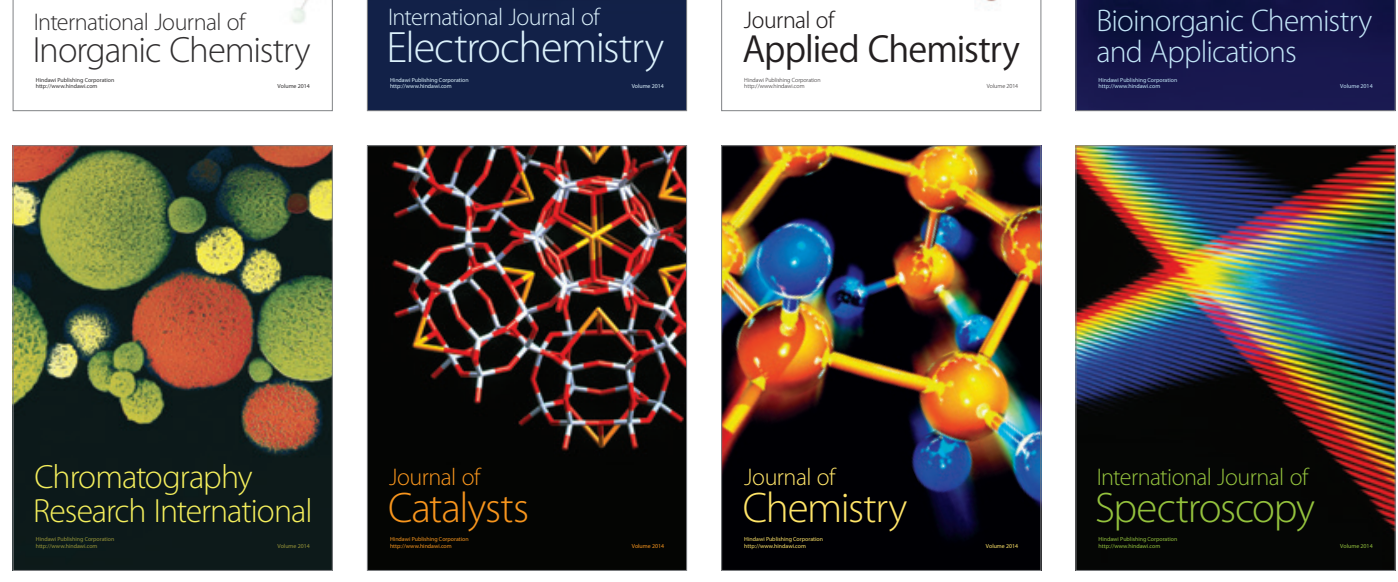